\title{
Full-field infrared phase sensitive thermography for microstructural investigation of giant magnetostrictive materials
}

\author{
Peng Yang, Chiu T. Law and Rani F. Elhajjar
}

\begin{abstract}
Background: Giant magnetostrictive materials are increasingly proposed for smart material applications such as in sensors, actuators, and energy harvesting applications. However, reviewing the literature on this topic, the reader observes a large amount of variability in the reported properties that are typically generated from overall strain or point-value strain measurements obtained with strain gages using the far field estimate to project the internal magnetic field in the specimen.

Methods: A full-field phase-sensitive thermography method is proposed to correlate the full-field infrared measurements to changes in the microstructure induced by a cyclic magnetic field in a giant magnetostrictive alloy material.

Results: The results show the potential of the proposed method in rapidly uncovering the effects of geometry and defects on the magnetostrictive response. The results show responses at the microstructure level from both magnetocaloric and magnetostrictive effects.

Conclusions: The effects of the magnetostrictive material's microstructural spatial variability and the specimen geometry on the localized magnetostrictive response warrant serious considerations but so far have not received significant attention. The method proposed is capable of highlighting magneto-elastic coupling in the composite specimens using the cycle magnetic field.
\end{abstract}

Keywords: Magnetostriction, Nondestructive testing, Mechanical behavior, Magnetocaloric effect, Thermography, Electronic materials

\section{Background}

Magnetostrictive materials belong to a class of smart materials that have been proposed for magneto-responsive materials. Terfenol-D $\left(\mathrm{Te}_{0.3} \mathrm{Dy}_{0.7} \mathrm{Fe}_{2}\right)$ is a giant magnetostrictive material consisting of rare earth elements terbium, dysprosium and iron. The positive magnetostrictive response refers to the increase of the mechanical strain in the material as the magnitude of an external magnetic field is raised. A 'giant' magnetostrictive material, as opposed to just a magnetostrictive material, has a maximum strain usually in the hundreds part per million when saturated with a magnetic field. Terfenol-D was developed in the 1970s at

\footnotetext{
* Correspondence: elhajjar@uwm.edu

College of Engineering \& Applied Science, University of Wisconsin-Milwaukee, Milwaukee, WI, USA
}

the Naval Ordnance Laboratory which was used as a component in sonar transducers (Olabi and Grunwald 2008). This material is unique for its huge magnetostriction compared to other materials, such as Galfenol or nickel. This unique property has also led many proposals for its applications in actuation and energy harvesting (Ashley 1998; Goodfriend and Shoop 1992; Fenn et al. 1996). Magnetostriction can be attributed to the rotation of the magnetic moments for the alignment with the external magnetic field direction (Engdahl 2000). In Terfenol-D specifically, the large deformation results from the complementary magnetic anisotropies of terbium and dysprosium (Clark et al. 1984). The energy involved with magnetostriction is related to both the energy to deform the material and the magnetic field energy (Xe and Zheng 2005). A common issue in experiments 
with magnetostrictive material is the observation of large amount of scatterers. These can be attributed to the material quality, such as the number of defects. These alloys are capable of a large response in a wide range of operating temperatures (Clark 1992). Statistical analysis of the material properties can be accomplished with a coefficient of variation (CV) of approximately 5\% (Dapino et al. 2006). Higher temperatures can affect the saturation strain or the maximum strain under magnetic field stimulation (Clark and Crowder 1985). These materials also display anisotropic magnetostriction properties depending on the direction of the load and prestress application. The origins and the stress dependence of the magnetostrictive effect have been discussed by Bulte and Langman (Bulte and Langman 2002). The presented hypothesis is based on an atomic level theory of the origins of the magnetomechanical behaviour whereby spin-spin and spin-orbit coupling interact with magnetic moments to alter the magnetocrystalline anisotropy and exchange energies. Terfenol-D particles have a C15 cubic Laves crystal structure (contributing to high brittleness and hardness). This intermetallic phase has a polymorphic structure, meaning that it can change into different crystalline structures maintaining the same chemical composition (Alexander and Myers 2014). An enhanced magnetostriction is observed with Terfenol-D with higher ratios of the rare earth element $\mathrm{Tb} / \mathrm{Dy}$ ratio at the same volume fraction of $\mathrm{RFe}_{2}$ due to the increase of magnetocrystalline anisotropy, which is controlled by the $\mathrm{Tb} / \mathrm{Dy}$ ratio within a crystal (Kwon et al. 2005). To quantify the quality of the magnetostrictive material, a technique is needed for the estimation of the spatial variability in responses. Such a technique can potentially help identifying the origin for variations and most importantly enable radical changes in manufacturing procedures, resulting in enhanced responses and the ability in engineering magnetostrictive properties.

Here, we report the study of the magnetostriction in Terfenol-D under periodic variations of the magnetic field with phase sensitive thermography (PST) (Breitenstein et al. 2010). In this method, a lock-in amplifier infers the magnetic state with the thermal images with a reference signal. This technique is particularly useful for isolating small temperature variations from the intense background noise. A load (or magnetic field) is applied at a preset frequency enough to induce adiabatic conditions. As the specimen is loaded, an infrared camera captures these load related thermal changes and correlates them with a reference signal provided by the load machine (Greene et al. 2008). The advantage of this technique is in filtering camera noises and ambient temperature fluctuations. As a result, the detection of small temperature in the $\mathrm{mK}$ range becomes feasible. This thermography technique has been previously used in various engineering mechanics measurements (e.g., stress gradients) or defect evaluations when the thermoelastic effect is present (i.e., infrared signals related to sum of principal stresses) (Wu et al. 1994; Meola et al. 2006; Elhajjar et al. 2014; Haj-Ali et al. 2008). Here, PST is investigated for evaluating the fabrication quality of magnetostrictive materials.

During the alignment of the magnetic moments, thermal energy is released in the form of heat. This release of heat is also known as the magnetocaloric effect (MCE). The internal energy of the material system is at a high energy state which is the result of the energy input into the material system during its formation (Gomez et al. 2013). The applied magnetic field direction is a low energy state. When the magnetic moments align towards the magnetic field, the moments go from a high energy state to a lower energy state. Under adiabatic conditions, this change in energy is compensated through the release of heat in the material (Tishin and Spichkin 2003). In adiabatic conditions, the change of temperature due to the change of the magnetic field (or magnetocaloric effect), $\Delta T$ can be defined as (Tishin and Spichkin 2014):

$$
\Delta T=\int_{H_{1}}^{H_{2}} \frac{T}{C_{H}}\left(\frac{\partial M}{\partial T}\right)_{H} d H
$$

Where $C_{H}$ is the heat capacity under constant magnetic field, $H$ is the magnetic field strength, and $M$ is the magnetization. In the case of thermoelastic effect, under adiabatic conditions and applying Lamé elastic parameters which are assumed to be independent of temperature, we arrive at the classical theory of thermoelastic stress (Pitarresi and Patterson 2003), the change in temperature $\Delta T$ becomes:

$$
\Delta T=-\frac{\alpha}{\rho c_{p}} T_{o} \Delta\left(\sigma_{1}+\sigma_{2}\right)
$$

Where $\alpha$ is the coefficient of thermal expansion, $\rho$ is the density, $c_{p}$ is the specific heat capacity at constant pressure, $T_{o}$ is the initial temperature, and $\sigma_{1}$ and $\sigma_{2}$ are the first and second principal stresses, respectively.

\section{Methods}

The monolithic giant magnetostrictive material used in this study was a polycrystalline Terfenol-D from TdVib LLC (Ames, Iowa, USA) with its properties shown in Table 1. The test samples were machined from a larger piece and the final form was a rectangular bar (Table 2). The specimens tested were a large specimen (TL) and a small specimen (TS) as shown in Fig. 1. It should be noted that specimen (TL) contains side notches to study the effect of geometry. The surfaces of the samples were painted matte black using black paint (Ultra Flat, Krylon, USA). Black paint was used because it simulates a black body increasing its emissivity for the thermal camera. With a high emissivity of $94 \%$, the paint maintains this emissivity from point to 
Table 1 Properties of Terfenol-D (Terfenol-D 2015)

\begin{tabular}{lll}
\hline Terfenol D Physical Properties & & \\
\hline Mechanical Properties & 9200 & $\mathrm{~kg} / \mathrm{m}^{3}$ \\
Density & 90 & $\mathrm{GPa}$ \\
Bulk Modulus & $28-40$ & $\mathrm{MPa}$ \\
Tensile Strength & $300-880$ & $\mathrm{MPa}$ \\
Compressive Strength & & \\
Thermal Properties & 11 & $\mathrm{Ppm} /{ }^{\circ} \mathrm{C} @ 25^{\circ} \mathrm{C}$ \\
CTE & 330 & $\mathrm{~J} /(\mathrm{kg}-\mathrm{K})$ \\
Specific Heat & & $\mathrm{ppm}$ \\
Magnetostrictive Properties & $800-1200$ & $\mathrm{~nm} / \mathrm{A}$ \\
Strain & $6-10$ & \\
Piezomagnetic Constant & & \\
Magnetic Properties & $2-10$ & \\
Relative Permeability & & \\
\hline
\end{tabular}

point within $2 \%$ up to a view angle of around $20^{\circ}$ degrees from the surface (Oliver 1988). The paint layer thickness was measured at the test sample using a micrometer tool before and after the paint was applied at multiple locations. Paint layer thickness was measured to be approximately 10-20 $\mu \mathrm{m}$ uniformly across the specimen which corresponds to the opacity-limited region where there maybe coupling between the specimen and the coating. This is away from the region where the thick layer of paint can act as an insulating layer that can attenuate the thermal emission from the sample surface (Robinson et al. 2010).

The setup for PST technique is shown in Fig. 2. An Agilent 33120A function generator was set to generate a sinusoidal waveform at $2.5 \mathrm{~Hz}$, (1), and was fed into an $\mathrm{AE}$ Techron 7224 power amplifier, (2), which powered two large electromagnets, (3). The magnetostrictive sample, (4), was mounted between the two electromagnetic cores using a custom made 3D printed ABS clamp as shown in Fig. 3. A non-magnetic clamp was important for holding the specimen with a constant force. It also needed to be compact enough to be able to fit between two magnetic poles without disturbing the magnetic field. The clamp has a low profile and uses a single ply of plain weave carbon fiber epoxy composite as a leaf spring. The extreme bend in the spring allowed for a region of relatively constant applied force in its load-deflection profile. The low profile clamp allows for the two magnetic poles to be positioned close to form a narrower gap which is important

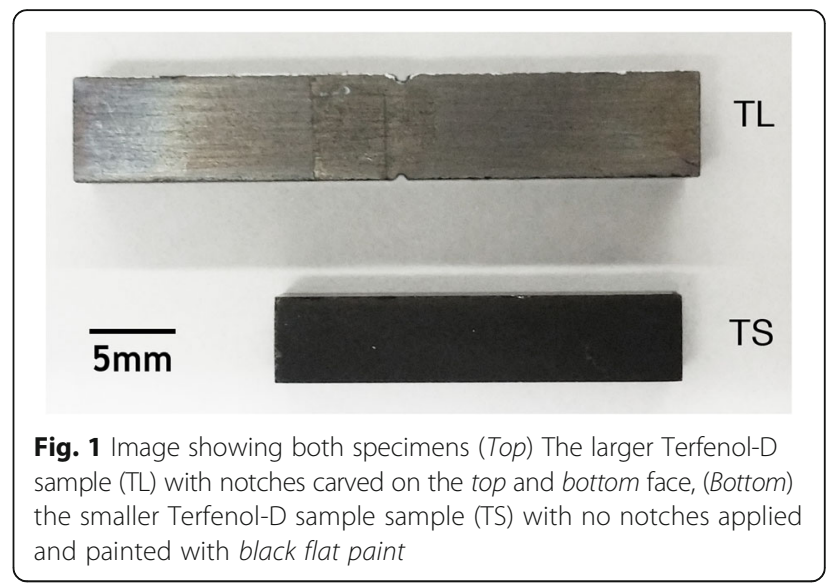

for achieving high magnetic fields. The sample was held in place with the clamp by applying a constant force of about $4.5 \mathrm{~N}$ to the sample. This was sufficient for preventing the sample from moving while allowing the sample to strain freely. This mechanism is achieved by having one end constrained while leaving the other end to displace accommodating material expansion. The applied force produced a negligible effect with pre-stress of less than $170 \mathrm{kPa}$. The clamp was constrained to a $12.7 \mathrm{~mm}$ diameter solid aluminum rod that was wedged between the two magnetic poles. The gap in the electromagnet was $56 \mathrm{~mm}$. The aluminum rod allowed for fine tuning of the positioning of the sample between the two cores. A Sypris SAD18-1904 gauss meter probe, (5), was mounted next to the sample's surface. The probe measured the magnetic field along its long axis and according to the tangential boundary condition, the measured magnetic field intensity relates to the field inside the specimen (Elhajjar and Law 2015). A Sypris Model 5180 gauss meter, (6), measures the magnetic field and outputs a signal at \pm 3 volts. The gauss meter signal was doubled in frequency with a rectifier, (7), to $5 \mathrm{~Hz}$ corresponding to the magnetostriction rate experienced by the sample. This signal was then recorded by the NI USB-6008 DAQ device, (8), and used as a reference signal. At the same time, a FLIR A35 microbolometer (9) collected infrared images at $60 \mathrm{~Hz}$. The infrared detector measures an un-calibrated PST signal so the measurements do not relate to absolute temperature calibrations. The captured infrared images from the camera and the reference signal were processed by the Microbolometer Thermoelastic Evaluation software, (10), to produce a single thermal image (Microbolometer Thermoelastic

Table 2 Characteristics of test specimens

\begin{tabular}{lllllll}
\hline Sample ID & Description & $\begin{array}{l}\text { Length, } \\
\mathrm{mm}\end{array}$ & $\begin{array}{l}\text { Width, } \\
\mathrm{mm}\end{array}$ & $\begin{array}{l}\text { Thickness, } \\
\mathrm{mm}\end{array}$ & $\begin{array}{l}\text { Mass, } \\
\mathrm{g}\end{array}$ & $\begin{array}{l}\text { Density, } \\
\mathrm{kg} / \mathrm{m}^{3}\end{array}$ \\
\hline TL & Lg. Monolithic Terfenol-D & 36.20 & 6.04 & 6.12 & 12.500 & 9341 \\
TS & Sm. Monolithic Terfenol-D & 23.68 & 5.00 & 4.98 & 5.404 & 9165 \\
\hline
\end{tabular}


(3)

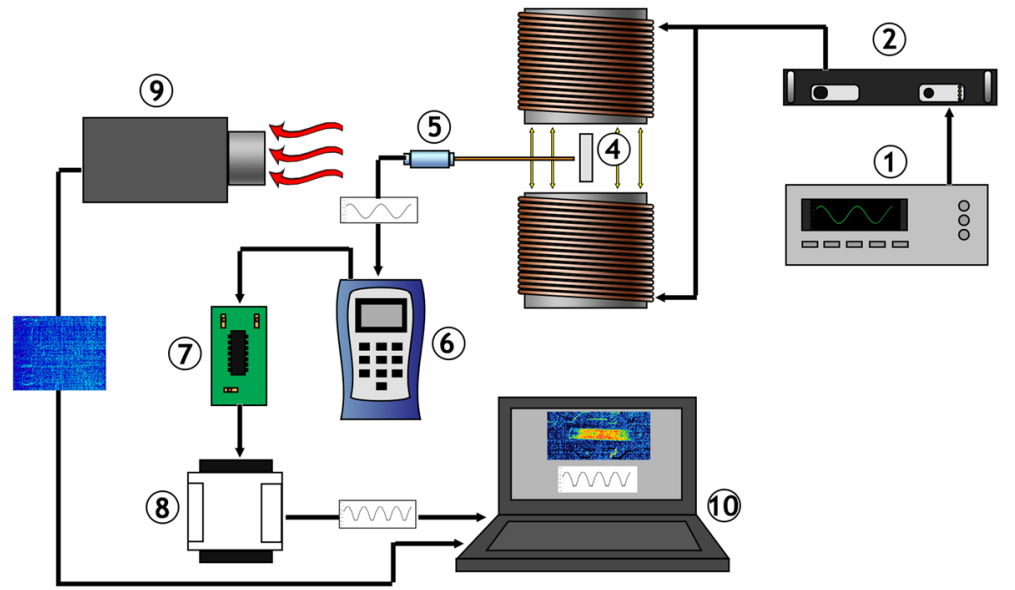

Fig. 2 Phase sensitive thermography test setup for giant magnetostrictive materials

Evaluation 2015). The TL sample was evaluated at six different magnetic field intensities while the TS sample was tested at five different intensities. To verify the results and ensure consistency, follow-up tests were performed by removing and reapplying the paint surface. The thermography tests were conducted at a room temperature of $295 \mathrm{~K}$. In addition to PST testing, independent strain measurements were also performed for the TL sample with a strain gage attached to the specimen as seen in Fig. 3. In this setup, the magnetic field was cycled at a rate of $0.2 \mathrm{~Hz}$ from 0 to $150 \mathrm{kA} / \mathrm{m}$ for several cycles to ensure consistency in strain measurement. The low cycle rate was used to prevent any introduction of noises to the strain gage from the changing field.

\section{Results and discussion}

The results from the PST method are shown in Fig. 4. The full-field infrared images are presented in the same orientation as the sample is shown in Fig. 3. The temperature change that was measured was very small in the order of a few $\mathrm{mK}$. From both the TS and TL samples, we have several observations. Figure 4 reveals the proportional increases in temperature with the increasing magnetic field and the non-uniformity of the temperature distribution throughout the sample surface. The top and bottom edges show higher temperature change versus the central horizontal axis. The right and left ends of the sample exhibit little to no thermal activity. Lastly, we noticed some artifactlike features that disrupt the pattern of the surface temperature gradient.

In the TL sample, a region of lower temperature exists near the middle of the sample. For the TS sample, the temperature of the bottom edge was much lower than that of the top edge. In addition, there was a discontinuity in the temperature gradient towards the right edge. These discontinuities were investigated by the applying and reapplying

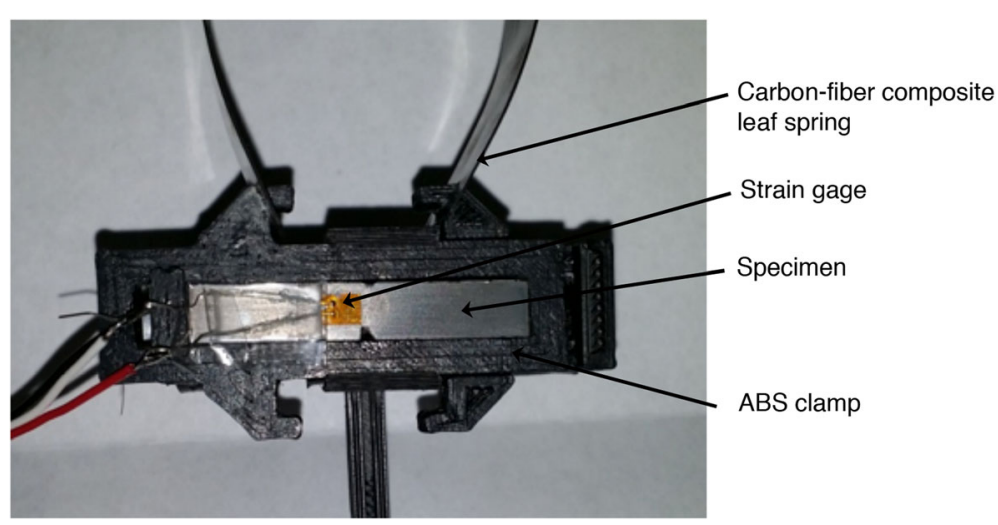

Fig. 3 Phase sensitive thermography test setup for giant magnetostrictive materials 


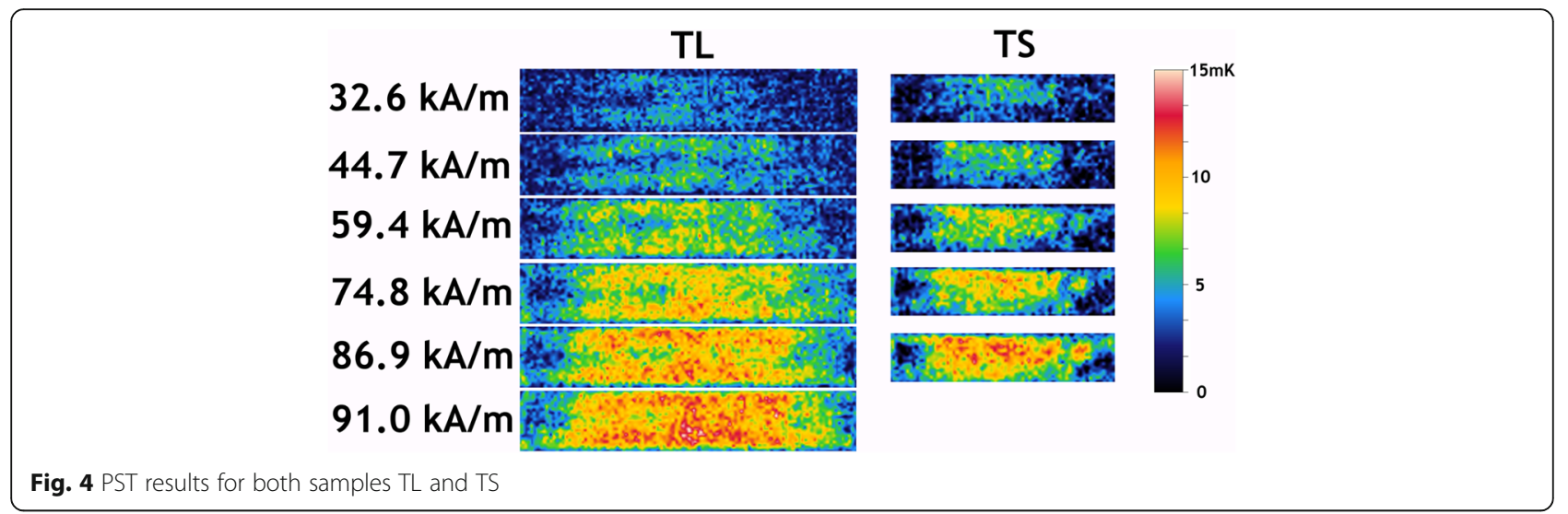

of the surface paint followed by rotation of the specimen. First, the test sample was tested with its original paint coating. The sample was then rotated by 180 degrees (or flipped upside down), to remove any bias that maybe associated with the thermal camera lens or test setup. Next the sample's paint surface was carefully removed and reapplied on to remove any effects that maybe caused by sample preparation. Paint thickness was measured after to ensure consistent thickness. The test was performed once again followed by the rotation of the specimen. The results of this investigation are shown in Figs. 5 and 6. The strain measurements and infrared images for the TL sample are both shown in Fig. 7. The graph shows the infrared images in relation to the measured strain values. It can be seen that from this graph that magnetostriction does not become saturated at the applied magnetic field. The results shown are in line with a simulation performed on a similar micromechanics composite system (Elhajjar and Law 2015). The results show that magnetization is linked to location and orientation of the Terfenol-D particles.
The test results reveal that increases of the amplitude of a cyclic magnetic field cause the PST signal response from the surface of the magnetostrictive material to raise. Such increase is likely attributed to magnetocaloric heating as the specimen was not restrained and was free to deform. However, if residual stresses were locked in the material, the thermoelastic effect maybe at play as the localized stresses are changed during the cyclic magnetic field application. It is not completely certain whether the MCE was at its maximum since this study was conducted at room temperature and to the best of authors' knowledge; the MCE of Terfenol-D has not been studied before. A study conducted by Nikitin et al. on Tb-Dy alloys of similar composition showed a maximum temperature change of $0.7 \mathrm{~K}$ when testing for MCE around $194 \mathrm{~K}$ (Nikitin et al. 1989). In another study conducted by Tereshina et al., a TbDyHoCo alloy was studied. When iron was added to the composition, the maximum temperature change dropped from $1.75 \mathrm{~K}$ to $0.5 \mathrm{~K}$. In addition, the temperature at which this greatest change occurs shifted from $140 \mathrm{~K}$ up to $280 \mathrm{~K}$. The addition of

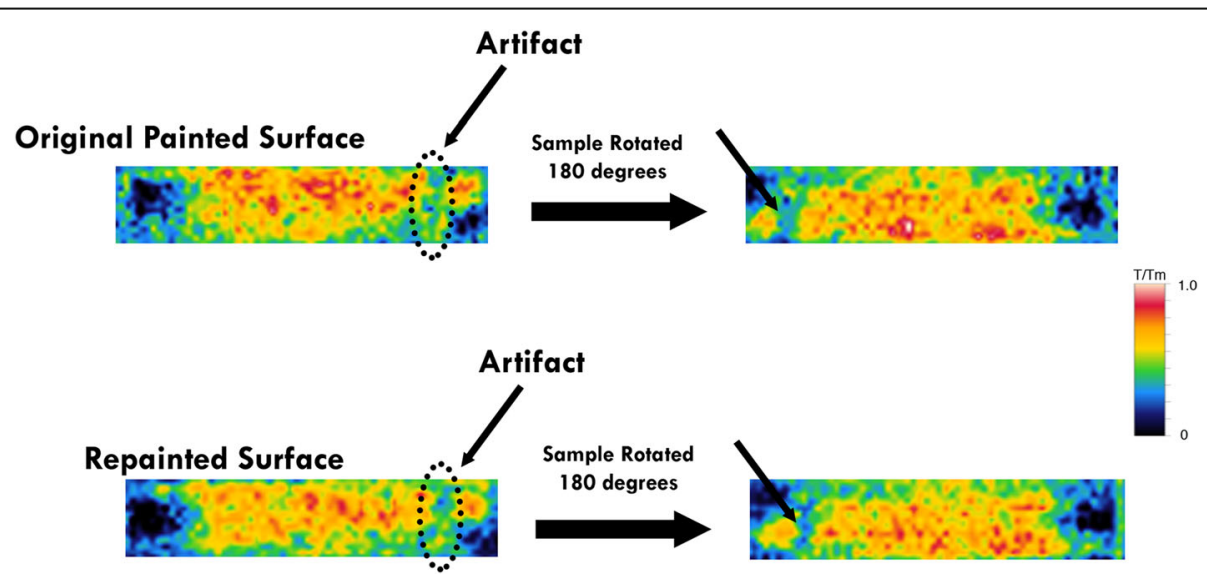

Fig. 5 Follow-up tests to investigate the variations seen the thermal images on surface of TS Specimen 


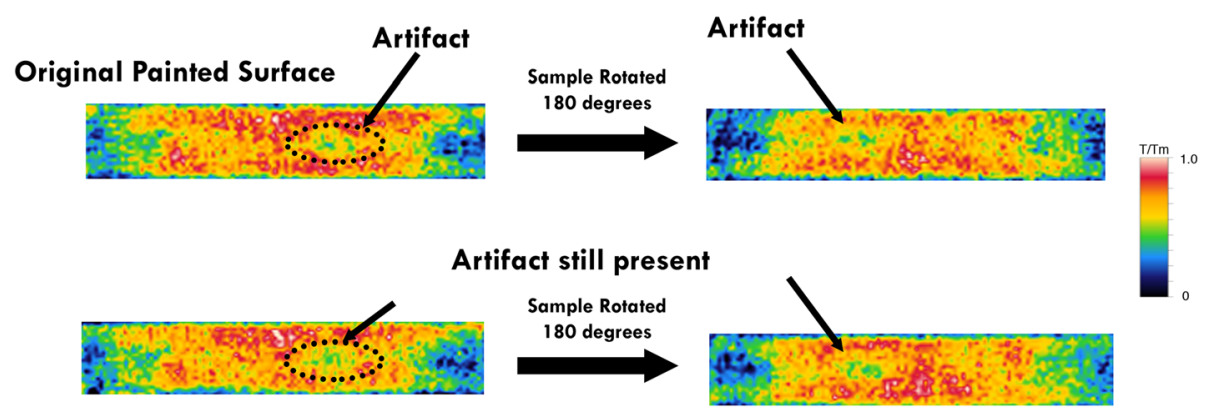

Fig. 6 Effect of paint and specimen rotation on observed thermal effects

the iron reduced the maximum change of temperature and shifts the operating temperature at which this peak occurs closer to room temperature. In the case of TbDy, we can expect the addition of iron to reduce the peak temperature change down to the values measured in this study. We can also expect the operating temperature at which this occurs to become closer to room temperature. The measured temperature change in this study was probably operating near the range of maximum MCE. Further study will need to be conducted to verify this.

Observation of the temperature gradient indicates that PST is sensitive to the geometric artifacts of the sample and their interactions with the magnetic field. The notches of the TL sample produced large PST signal at the central region. Edges and corners also produce higher PST signals that were seen along the top and bottom edge. The notch may concentrate the magnetic field in this region. This variation can be described by the third suggestion which is that full field PST can detect surface microstructural effects. The authors have performed detailed micromechanical simulation on similar composites that shows the region near the boundary have higher magnetization but that was also a function

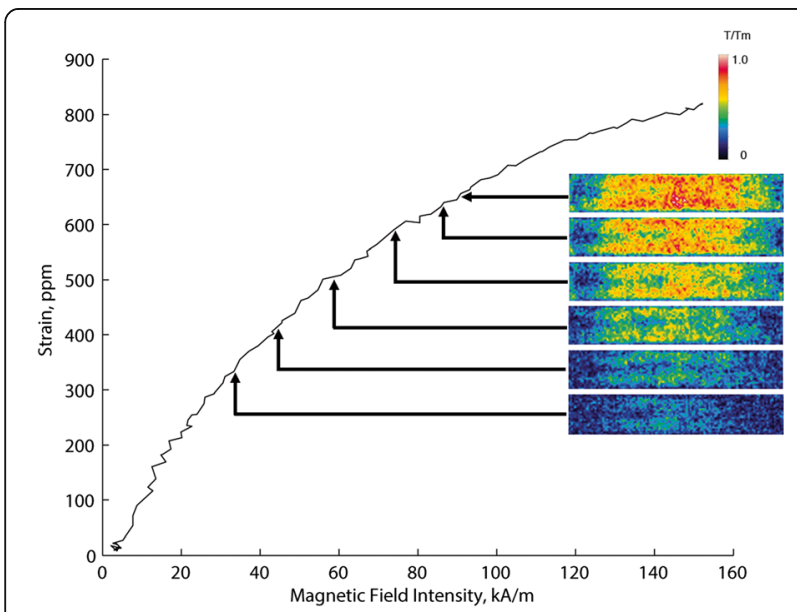

Fig. 7 Axial strain versus magnetic field intensity for specimen $T L$, insert shows change in temperature at various magnetic field strengths of the particle size and orientation (Elhajjar and Law 2015). This may explain the higher temperature changes at the edges. The lack of resolution of the response around the notches is attributed to two factors. 1) The material inhomogeneity is a large factor in the response (i.e., not all parts of the material have the same response), ii) The resolution of the microbolometer limited the resolution. An infrared camera with a better spatial resolution would have helped better define this region.

The follow-up test into the artifact-like features revealed that these variations in temperature and discontinuities remain present. If these variations were due to experimental setup, the surface temperature profile should have changed when it was rotated. If the variations were due to sample preparation, then the surface temperature profile would also have changed. The follow-up test showed that the surface temperature profile remained constant leading to the conclusion that these variations are the result of a microstructural defect in the specimen.

\section{Conclusions}

Full field PST has the potential as a tool for the nondestructive evaluation of the microstructure of magnetostrictive materials. The PST signal from the infrared measurements from our investigations reveals a magnetocaloric effect in Terfenol-D and magnetic interactions with microstructural defects. The observed PST variations were consistent with the expectation for the polycrystalline material used in this study. The PST signal was sensitive to the sample geometry which causes magnetic boundary effects and potentially intensifying magnetic field intensity leading to strong PST signal fluctuations near notches and edges. The proposed full-field technique is adequately sensitive for detection of material inhomogeneity or design verification of a nanoor microstructured magnetostrictive transducers or actuators. Although there are little to no information on data pertaining to the MCE effect of Terfenol-D, the proposed method provides insight for the MCE 
effect in magnetostrictive materials, besides demonstrating ability in detecting geometry or material variability in magnetostrictive materials and devices. In addition to absence of standard methods for characterizing properties of magnetostrictive materials, it is a possibility that previously reported variability in magnetostrictive properties is from production methods of these polycrystalline materials that do not yield consistent properties. The ratios of rare earth metals in the alloy may not be uniformly distributed. However, geometrical effects and magnetocaloric effects are also involved as well. Future research will need to address how to decouple the thermal response to isolate the effects from the mechanical stresses between crystals produced by the magnetostriction and the magnetocaloric effect due to alignment of magnetic moments, both of which contribute to the observed thermal changes. Separating the two factors contributing to the temperature changes can allow the ability to understand the relative contribution of each component. This can perhaps be accomplished by measuring the strain simultaneously using a full-field method such as digital image correlation to correlate the regions of high strain with the temperature values measured. Understanding these sources of variability on the magnetostrictive response can lead to more accurate property characterizations and more precise engineering of magnetostrictive actuators and sensors.

\section{Abbreviations}

MCE: Magneto Caloric Effect; PST: Phase Sensitive Thermography; TL: Terfenol-D Large Specimen; TS: Terfenol-D Small Specimen

\section{Acknowledgements}

The Engineering Mechanics and Composites Research laboratory for providing some of the test fixtures for this research.

\section{Funding}

The authors would like to thank the University of Wisconsin-Milwaukee Research Growth Initiative for their support in this research.

\section{Availability of data and materials}

All datasets will be provided on the University of Wisconsin-Milwaukee (UWM) Digital Commons open access repository. It is a service of the University of Wisconsin-Milwaukee Libraries, is a virtual showcase for UWM's research and creative profiles. UWM is part of global Digital Commons and ensures for long-term preservation and worldwide electronic accessibility.

\section{Authors' contributions}

R.E. and C.L. conceived the idea of this research. Design of test setup and execution of testing was performed by P.Y. Analysis of test results and writing this manuscript was performed by all the authors. All authors read and approved the final manuscript.

\section{Author's information}

PY is a graduate student of Materials Science and Engineering at the University of Wisconsin-Milwaukee. He completed his B.S. in Mechanical Engineering. CL is associate professor in Electrical Engineering and is interested in the use of magnetostrictive materials in sensor applications. RE is associate professor in the Civil and Materials engineering department. He is interested in magnetostrictive materials for application in composite materials for smart material applications.

\section{Competing interests}

The Authors declare that there is no conflict of interest involved in this submission.

\section{Publisher's Note}

Springer Nature remains neutral with regard to jurisdictional claims in published maps and institutional affiliations.

Received: 23 December 2016 Accepted: 15 May 2017

Published online: 25 May 2017

\section{References}

Alexander JH, Myers OJ (2014) Microstructure Properties and Strengthening Mechanisms of the AS4-3501-6 Polymeric Resin With Embedded Terfenol-D Particles. In: ASME 2014 Conference on Smart Materials, Adaptive Structures and Intelligent Systems, C. American Society of Mechanical Engineers, Newport, pp V001T005A014-V001T005A014

Ashley S (1998) Magnetostrictive actuators. Mechanical Engineering, vol 120

Breitenstein O, Warta W, Langenkamp M (2010) Lock-in Thermography: Basics and Use for Evaluating Electronic Devices and Materials. 2nd edn. Springer, Berlin

Bulte DP, Langman RA, 2 (2002) Origins of the magnetomechanical effect. J Magn Magn Mater 251:229-243. doi:10.1016/S0304-8853(02)00588-7

Clark AE (1992) Giant magnetostriction materials from cryogenic temperatures to $250^{\circ} \mathrm{C}$, San Diego-DL tentative, International Society for Optics and Photonics. pp 374-381

Clark A, Crowder D (1985) High temperature magnetostriction of TbFe 2 and Tb. 27 Dy. 73 Fe 2. Magnetics, IEEE Trans 21(5):1945-1947

Clark AE, Savage HT, Spano ML (1984) Effect of stress on the magnetostriction and magnetization of single crystal Tb. 0.27 Dy. 73 Fe 2. IEEE Trans Magn 20(5):1443-5

Dapino MJ, Flatau AB, Calkins FT (2006) Statistical analysis of Terfenol-D material properties. J Intell Mater Syst Struct 17(7):587-599

Elhajjar RF, Law CT (2015) Magnetomechanical local-global effects in magnetostrictive composite materials. Modeling and Simulation in Materials Science and Engineering, vol 23

Elhajjar R, Haj-Ali R, Wei B-S (2014) An Infrared Thermoelastic Stress Analysis Investigation for Detecting Fiber Waviness in Composite Structures. Polym-Plast Technol Eng 53(12):1251-1258

Engdahl G (2000) Handbook of Giant Magnetostrictive Materials. Academic, San Diego, p 92101

Fenn RC, Downer JR, Bushko DA, Gondhalekar V, Ham ND (1996) Terfenol-D driven flaps for helicopter vibration reduction. Smart Material Structures, vol 5

Gomez JR, Garcia RF, Catoira ADM, Gomez MR (2013) Magnetocaloric effect: A review of the thermodynamic cycles in magnetic refrigeration. Renewable and Sustainable Energy Reviews, vol 17

Goodfriend MJ, Shoop KM, 2 (1992) Adaptive characteristics of the magnetostrictive alloy, Terfenol-D, for active vibration control. J Intell Mater Syst Struct 3:245-254

Greene RJ, Patterson EA, Rowlands RE (2008) Thermoelastic Stress Analysis. In: Sharpe WN (ed) Handbook of Experimental Solid Mechanics. Springer, Berlin, pp 743-763

Haj-Ali R, Wei BS, Johnson S, El-Hajjar R (2008) Thermoelastic and infraredthermography methods for surface strains in cracked orthotropic composite materials. Eng Fract Mech 75(1):58-75

Kwon OY, Kimb JC, Kwon YD, Yang DJ, Lee SH, Lee ZH (2005) Magnetostrictive properties of TbxDy1-xFey $(x=0.3,0.33 ; y=1.05,1.36,1.65)$ composite made by directional solidification and polymer infiltration. In: Zhong ZY, Saka H, Kim TH, Holm EA, Han YF, Xie XS (eds) Pricm 5: The Fifth Pacific Rim International Conference on Advanced Materials and Processing, Pts 1-5. Mater Sci Forum 475-479:2247-2250

Meola C, Carlomagno GM, Squillace A, Vitiello A (2006) Non-destructive evaluation of aerospace materials with lock-in thermography. Engineering Failure Analysis, vol 13

Microbolometer Thermoelastic Evaluation (2015) Australian Government Department of Defense, Science and Technology, 12th edn

Nikitin SA, Tishin AM, Leontev PI (1989) Magnetocaloric effect and refrigerant capacity of Tb-Dy alloys. Physica Status Solidi, vol 113

Olabi A, Grunwald A (2008) Design and application of magnetostrictive materials. Materials and Design, vol 29

Oliver DE (1988) Stress pattern analysis by thermal emission (SPATE). Exper Tech; (United States) 12 
Pitarresi G, Patterson EA (2003) A review of the general theory of thermoelastic stress analysis. The Journal of Strain Analysis for Engineering Design, vol 38 Robinson AF, Dulieu-Barton JM, Quinn S, Burguete RL (2010) Paint coating characterization for thermoelastic stress analysis of metallic materials. Measurement Science and Technology, vol 11

Terfenol-D (2015) Etrema., http://www.etrema.com/. Accessed November 2015

Tishin AM, Spichkin YI (2003) The Magneteocaloric Effect and Its Applications. Institute of Physics Publishing, Series in Condensed Matter Physics

Tishin A, Spichkin Y (2014) Recent progress in magnetocaloric effect: Mechanisms and potential applications. Int J Refrig 37:223-229

Wu D, Karben W, Haupt K, Walther HG, Busse G (1994) Applications of phase sensitive thermography for nondestructive evaluation. J Phys IV 4(C7):C7-C567

Xe L, Zheng X (2005) A nonlinear constitutive model for magnetostrictive materials. Acta Mechanica Sinica, vol 21

\section{Submit your manuscript to a SpringerOpen ${ }^{\circ}$ journal and benefit from:}

- Convenient online submission

Rigorous peer review

- Open access: articles freely available online

- High visibility within the field

Retaining the copyright to your article

Submit your next manuscript at $>$ springeropen.com 